\title{
Une gestion professionnelle du foyer : un récit de genre pour les lectrices de Marie-Claire (1954-1955)
}

Household management : a gender narrative for Marie-Claire's readers (1954-1955)

\section{Alexie Geers}

\section{OpenEdition}

\section{Journals}

Édition électronique

URL : http://journals.openedition.org/itti/711

DOI : 10.4000/itti. 711

Éditeur

Université de Poitiers

Référence électronique

Alexie Geers, «Une gestion professionnelle du foyer : un récit de genre pour les lectrices de MarieClaire (1954-1955) », Images du travail, travail des images [En ligne], 6-7 | 2019, mis en ligne le 01 février 2019, consulté le 14 avril 2021. URL : http://journals.openedition.org/itti/711 ; DOI : https://doi.org/ 10.4000/itti.711

Ce document a été généré automatiquement le 14 avril 2021.

Images du travail, travail des images 


\title{
Une gestion professionnelle $\mathrm{du}$ foyer : un récit de genre pour les lectrices de Marie-Claire (1954-1955)
}

\author{
Household management : a gender narrative for Marie-Claire's readers
}

(1954-1955)

\section{Alexie Geers}

1 Le 5 mars 1937, le magazine Marie-Claire paraît pour la première fois avec pour objectif de « donner à la femme française, en un seul journal, chaque semaine, tout ce qui peut l'intéresser ou lui être utile ${ }^{1} »$. Ce projet est soumis par Marcelle Auclair, jeune journaliste, à Jean Prouvost, propriétaire de presse, pour combler un vide éditorial : elle estime que les femmes, pensées comme catégorie unie par le sexe, n'ont pas de magazine pour répondre à leurs besoins de divertissement et d'informations. Ils réalisent cet objectif en diffusant des contenus éditoriaux variés et en mettant à disposition des espaces publicitaires aux annonceurs travaillant au service des industriels qui souhaitent promouvoir leur production auprès des femmes. Rédaction et annonceurs mobilisent, tous deux, textes et images en nombre dans une formule qui reçoit dès ses débuts un accueil très favorable.

2 Il me semble que l'analyse des choix de sujets et de la manière dont ils sont traités, mis en mots et en images dans ces contextes, permet de décrire des " récits de genre ", soit des ensembles de savoirs, de pratiques ou de justifications définissant des rôles genrés (Geers, 2016). Pendant les premières années de publication de Marie-Claire, le modèle de féminité est principalement fondé sur le concept de beauté et invite les femmes à travailler leur apparence et affirmer leur pouvoir de séduction pour construire le bonheur amoureux au sein du couple. Cet objectif domine largement toutes les autres activités "féminines » abordées dans le titre, et notamment celles liées au foyer qui sont alors décrites brièvement. Ce modèle vaut jusqu'en 1944, date à laquelle la publication du magazine est interdite, comme tous les titres de presse ayant continué de paraître sous l'Occupation. 
3 En octobre 1954, cependant, quand Marie-Claire reparaît après dix années de suspension, une place majeure est donnée à la gestion du foyer. Ce rôle est alors décrit comme un métier et reprend des éléments rhétoriques qu'avait développés Paulette Bernège, la fondatrice de la ligue d'organisation ménagère (1924) et du concept de 《rationalisation domestique $»^{2}$ pendant l'entre-deux guerres à destination de la bourgeoisie. À cette période, la responsabilité des épouses évolue (Martin, 1987), suite, entre autres choses, à une "crise des domestiques: jusqu'alors elles géraient le personnel de maison pendant que les maris s'occupaient de faire fructifier la rente, selon une division socio-sexuée des activités et des sphères (Kergoat, 2005), elles doivent désormais s'occuper plus pratiquement de la maison. Cette fonction, de « reines du foyer » (Bard, 2003), s'appuie, par ailleurs, sur l'émergence de l'industrie de l'électroménager et du mobilier en série (Delaunay, 2003) et tend à promouvoir ces productions auprès d'un public qui a les moyens de les acquérir et s'équipe au Salon des Arts Ménagers (Leymonerie, 2006). En valorisant les savoirs nécessaires à cette fonction, en faisant référence au monde de l'entreprise et en dessinant une figure de gestionnaire de la maisonnée, Paulette Bernège décrit à ces femmes aisées un rôle de genre positif (Clarke, 2005) .

4 Mon hypothèse est que ce modèle de féminité, basé sur l'investissement dans la sphère du foyer, dédié aux lectrices de Marie-Claire à partir de 1954, permet de diffuser une production industrielle en pleine expansion mais également un rôle genré qui a du sens auprès des femmes des classes moyennes. Cette analyse revient sur la construction narrative, visuelle et symbolique de ce modèle dans les numéros de Marie-Claire, publiés sur deux années, en 1954 et 1955 . Cette période constitue un moment de réélaboration du titre après dix années de suspension: la rédaction assoit son positionnement dans un paysage éditorial qui a évolué depuis la fin de la guerre avec l'établissement de plusieurs titres importants, tels que Marie-France (1944) et Elle (1945). Ce travail s'inscrit dans la continuité de ceux qui décrivent la complexité des fonctions de la presse féminine et en proposent une démarche compréhensive (Eck, Blandin, 2010) auquel s'ajoute une prise en compte centrale des images.

\section{Apprendre à gérer son foyer à l'heure du progrès : un nouveau métier}

5 En 1937, à la création de Marie-Claire, la rédaction transmet quelques informations et conseils au sujet de l'entretien de la maison ${ }^{4}$, la décoration ${ }^{5}$ ou la cuisine ${ }^{6}$ mais ne développe ni ne justifie le rôle des femmes dans ces domaines. À partir d'octobre 1954, en revanche, la situation est différente : de nombreux articles et publicités sont à la fois consacrés à la décoration et à l'aménagement du logement, à l'alimentation de la famille et à l'éducation des enfants, au «foyer » donc, et accompagnés d'une narration complexe qui rapproche la responsabilité des femmes dans le foyer, d'un métier. Le terme désigne alors aussi bien le logement que ses habitant-e-s.

6 Ainsi, leur rôle est décrit comme une activité professionnelle qui repose sur des connaissances spécifiques qu'elles doivent acquérir. Du côté du logement, les lectrices sont invitées à explorer plusieurs domaines comme l'aménagement de l'espace, la maîtrise de l'éclairage ou la composition des nouveaux matériaux (tissu, revêtement de sol, etc.). Des «spécialistes qui connaissent à fond les ressources de toutes les techniques $»^{7}-$ architectes, décorateur-rice-s, éclairagistes - transmettent ces 
connaissances au cœur d'une rubrique intitulée "l'Équipe 54 » et développent des grands principes d'aménagement. Dans ces articles, ils décrivent également l'électroménager et le mobilier en série promus dans les publicités diffusées dans les pages du magazine.

Ces changements de pratiques doivent permettre de gagner en praticité, d'économiser temps et argent, et davantage encore, si celles-ci doivent être assurées en dehors des heures ouvrées, comme l'indique un article de janvier 1955 :

«Cette cuisine est le domaine de Françoise, c'est aussi le souci $\mathrm{n}^{\circ} 1$ de toutes les jeunes maîtresses de maison qui travaillent et qui doivent en dix minutes préparer un repas $»^{8}$.

8 Pour autant, le travail des femmes en dehors de la sphère domestique est, généralement, présenté comme secondaire dans la vie de ces dernières, derrière les occupations liées aux soins de la famille. Dans une enquête, publiée en novembre 1954, dans la rubrique " Nous les jeunes ", à la question " considérez-vous que la femme doit travailler pour améliorer le budget du ménage?», trois personnes répondent positivement ( 2 femmes et 1 homme) et seulement une personne (homme) négativement. Cependant quatre personnes ajoutent «oui tant qu'elle n'a pas d'enfants » (2 hommes, 2 femmes).

sa famille prime et les femmes ont pour mission de lui composer un intérieur plus agréable. Le confort, proposé par les nouvelles industries, vient à point car la construction de nouveaux logements, collectifs notamment, équipés en eau courante et électricité est d'actualité et fournit les conditions nécessaires pour l'utilisation des nouvelles machines que sont le réfrigérateur ou la machine à laver (Delaunay, 2003).

Ces savoirs ne sont pas simplement égrainés mais accompagnés d'un discours élaboré sur la modernité qu'incarnent les nouvelles technologies qui permettent «d'adapter vos maisons à votre époque $»^{10}$. Cet argument est à mettre en relation avec un contexte national de développement industriel. Après la Seconde Guerre mondiale, l'argent de la reconstruction, issu du plan Marshall, est investi dans la modernisation de l'industrie (Woronoff, 1998). La production industrielle croitt pour atteindre, dès 1952, un bon niveau, plus particulièrement dans le secteur de l'électroménager et du mobilier en série, qui devient de fait, de plus en plus abordable (Delaunay, 2003). Symboliquement, la modernité industrielle est une référence positive car synonyme d'amélioration du quotidien et de progrès pour des hommes et des femmes qui voient leur niveau de vie progresser avec la mise en place du Salaire Minimum Interprofessionnel Garanti par exemple (Woronoff, 1998).

11 Les connaissances à acquérir concernent également les membres du foyer et plus spécifiquement les enfants : leur alimentation et leur éducation. La rédaction vulgarise des savoirs scientifiques nouveaux dans le domaine de la diététique ou encore de l'hygiène, et notamment dans une nouvelle rubrique de plusieurs double-pages consacrée à la puériculture et intitulée «Claude Maman». Une jeune journaliste enceinte, Claude, partage conseils puis expériences sur la grossesse, la naissance et l'éducation des enfants. Sur la question alimentaire, est indiquée, par exemple, aux mères la manière de "préparer chaque soir un savoureux cocktail de vitamines » aux enfants qui déjeunent à la cantine le midi ${ }^{11}$, en choisissant les bons aliments dans les bonnes proportions. L'adéquation entre ces nouvelles attentes en matière de santé et la production industrielle, vantée dans les publicités, est alors soulignée comme lorsque Claude raconte qu'elle a commencé par allaiter puis a rapidement donné du lait en 
poudre à ses jumelles qui «ne s'en portent pas plus mal »" Dans ce domaine également, l'idée de modernité est mobilisée et est associée à celle de sécurité lorsqu'il s'agit de promouvoir la production issue des industries agro-alimentaires. En février 1955 , les biscuiteries Lu sont présentées ${ }^{13}$ comme des «installations ultra-modernes ». Il est fait, par ailleurs, référence au gain de temps que permet l'usage de ces produits industriels : dans la publicité Royco publiée en février 1955: «Vite à table, c'est du Royco $»^{14}$.

Du côté de l'éducation des enfants, les savoirs diffusés sont issus des recherches récentes en psychologie du développement. Les travaux de référence comme ceux de Jean Piaget sur les capacités intellectuelles précoces des jeunes enfants ou ceux d'Henry Wallon consacrés à l'influence de l'environnement sur le bon développement des nourrissons sont perceptibles. Les mères sont invitées à faire une place centrale aux enfants dans la famille et à prendre en compte, davantage qu'auparavant, leur personnalité ${ }^{15}$ mais également, sur ce thème encore, à s'équiper d'objets industriels facilitant leurs soins (mobilier pour enfant, jouets d'éveil, etc.). Cette actualisation des pratiques liées aux enfants est décrite comme une manière d'améliorer leur éducation et de favoriser les liens au sein de la famille, institution particulièrement valorisée durant ces années dans le magazine.

13 L'investissement des femmes dans la famille s'inscrit dans un contexte politique qui invite à la procréation et au repeuplement du pays. L'expansion de l'allocation familiale, la réduction d'imposition des foyers avec enfants, l'allocation de salaire unique versée aux couples dont les femmes ne travaillent pas et l'assurance maternité (Martin J., 1998) composent une politique familiale offensive. Le montant de ces aides peut, d'ailleurs, dépasser le salaire d'une ouvrière lorsque cette dernière a trois enfants (Thébaud, 2001). Ces valorisations, bien qu'elles soient symboliques, permettent aux femmes d'exercer un rôle dans la sphère publique en participant à la recomposition de la société d'après-guerre (Knibiehler, 1997).

D'ailleurs, le rôle décrit n'est pas celui d'une simple exécutante mais celui d'une responsable, qui doit par exemple, travailler à la gestion financière du foyer. Cela apparaît, par exemple, dans l'argumentation développée autour du prêt bancaire ou dans l'article «Le crédit vous permet " publié en octobre 1954. Le foyer est assimilé à une entreprise dont les femmes doivent améliorer la cadence, faisant référence aux principes du taylorisme :

«Pourquoi modernise-t-on les houillères? Pour accroître le rendement des mineurs. De même on pourrait améliorer sérieusement le rendement des ménagères en modernisant nos installations (...) Le crédit vous permet (...) de réaliser des économies de temps et d'argent (...) de profiter immédiatement des progrès techniques ${ }^{16}$."

15 La présence de ce type d'argumentaire est un indicateur pour identifier le public visé dans le journal. Les lectrices ciblées ont un niveau de vie suffisant pour emprunter de l'argent mais non pour acheter comptant puisque le crédit est présenté comme un moyen de posséder davantage et de gravir l'échelle sociale :

«Je sais bien qu'en France le crédit n'est pas encore entré dans le mœurs. Pourquoi? parce que les Français s'imaginent que recourir au crédit, c'est se déclasser. Le crédit croit-on, est presque synonyme de pauvreté. En réalité, le crédit accroit, bien loin de le diminuer, votre standing ${ }^{17}$. »

16 L'acquisition d'une production industrielle innovante et du mode de vie qui l'accompagne est un levier d'ascension sociale. Cet investissement financier et 
symbolique dans le foyer est perceptible dans le succès populaire que reçoit le Salon des Arts Ménagers à cette époque (Le Wita, Segalen, 1993). Après avoir été suspendu pendant la Seconde Guerre mondiale, il ouvre de nouveau en février 1948. Il accueille 1 million de visiteurs en 1950 et 1,4 millions en 1956.

Dans les numéros de 1954 et 1955, rédaction et publicitaires proposent une image de la féminité qui repose sur le rôle des femmes dans le foyer considéré comme un métier, à responsabilités, basé sur des connaissances techniques. Ce discours valorise et ancre dans la modernité un rôle de genre qui donne aux épouses et aux mères une responsabilité dans l'ascension sociale de la famille mais également dans la refonte de la société française d'après-guerre.

\section{Représenter les femmes, au travail... domestique}

$\mathrm{Au}$ cours de ces premières années de reparution, le parallèle entre le foyer et le monde professionnel passe également par la construction d'imageries complexes qui synthétisent des registres visuels hétérodoxes, dont l'agencement fait sens.

Dans les publicités pour l'électroménager, plus particulièrement mettant en évidence leurs qualités techniques, les images élaborées font référence au dessin industriel. Les différents composants des appareils sont, par exemple, représentés «en coupe " comme dans la publicité Sauter publiée en octobre 1954 ou Thomson publiée en février 1955.

Image 1. Page 47, Publicité Sauter, Marie-Claire, n 1, octobre 1954

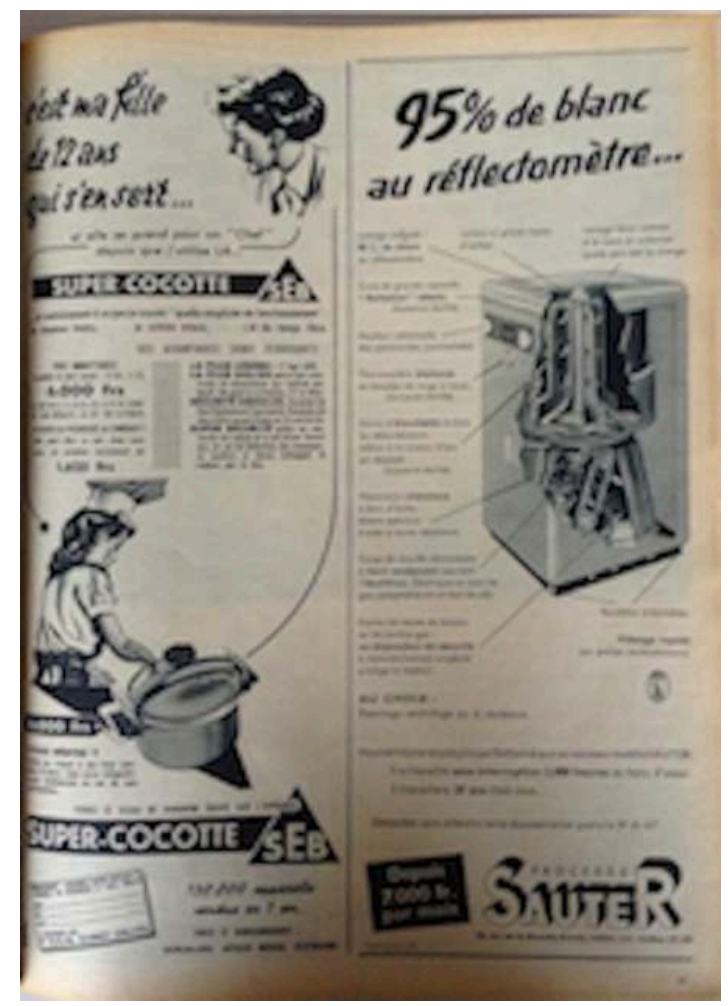

Coll. Musée Galliera 
Image 2. Page 86, Publicité Thomson, Marie-Claire, n 5, février 1955

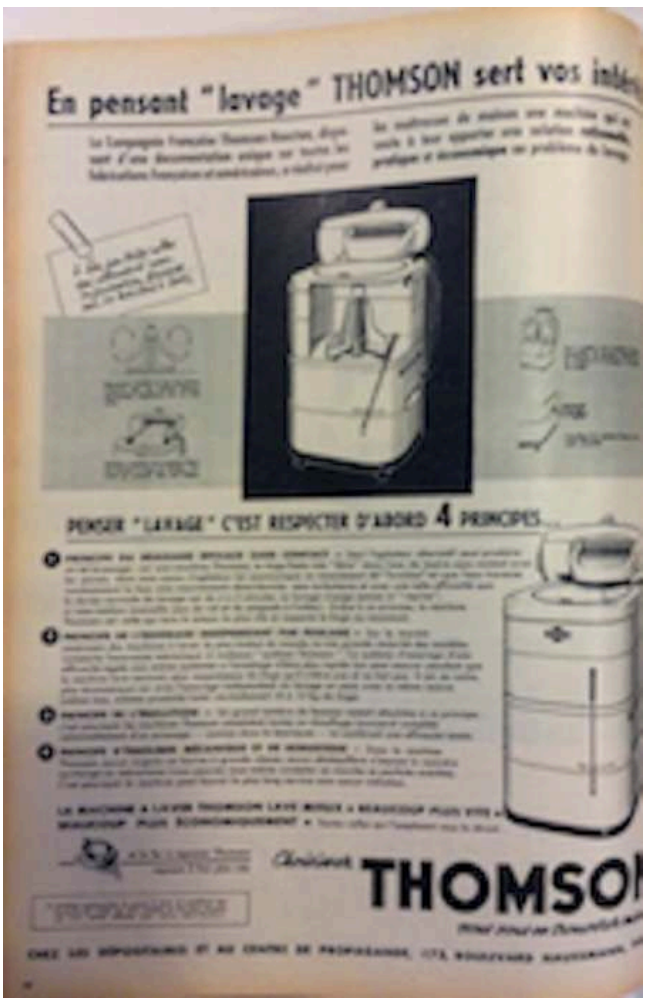

Coll. Musée Galliera

Dans la première, la cuve de la machine à laver est partiellement effacée pour laisser apparaître le mécanisme complexe qu'elle cache. Dans la seconde, plusieurs images accompagnent la description de la machine: une vue extérieure la montre dans son entier, une seconde en coupe partielle, les autres, plus petites, décrivent les détails du mécanisme comme l'hélice, élément clé du fonctionnement de la machine à laver. Les différents éléments peuvent également être représentés en gros plan et/ou vue éclatée ${ }^{18}$ comme dans la publicité Frigidaire. 
Image 3. Page 113, Publicité Frigidaire, Marie-Claire, n 3, décembre 1954

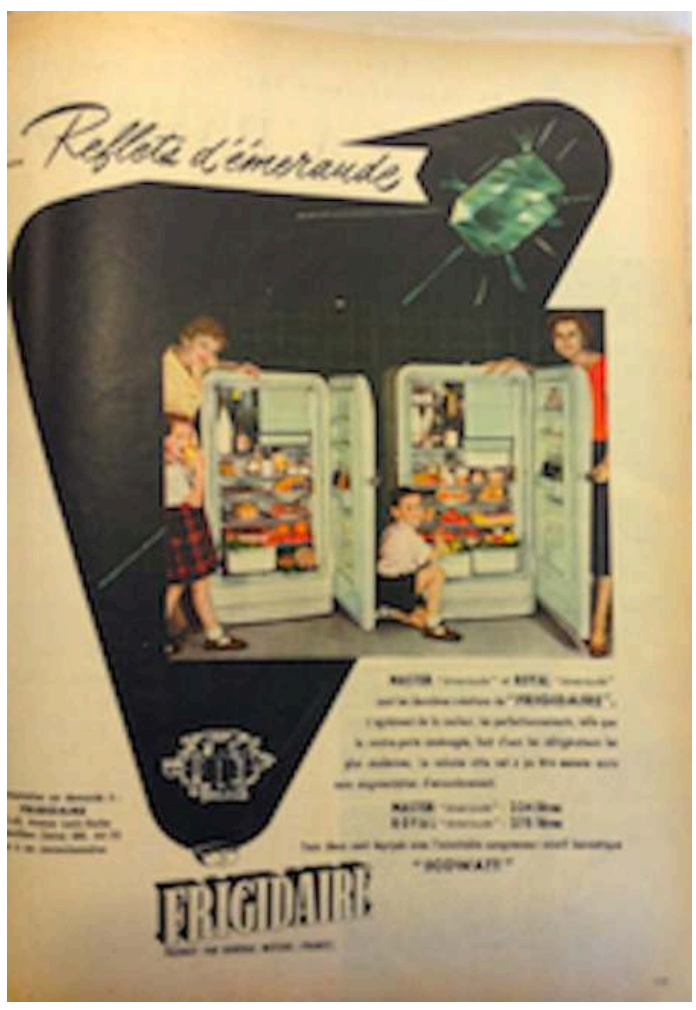

Coll. Musée Galliera

21 Sur cette dernière, en dessous d'une photographie montrant deux femmes qui ouvrent leurs réfrigérateurs pour révéler son importante capacité de contenance, «l'inimitable compresseur rotatif hermétique Ecowatt » est dessiné en gros plan. La représentation de cette pièce met en valeur sa technicité et son caractère innovant. En rappelant que le Frigidaire est "produit par General Motors France ", fabricant de voiture solide et réputé, le sérieux de la fabrication est assuré.

Cette mobilisation du monde industriel est renforcée par le recours à un vocabulaire spécialisé rappelant que le métier de gestionnaire du foyer nécessite des savoirs techniques et par là un apprentissage, comme toute activité professionnelle. Dans la publicité Conord, chaque pièce de ce rouage est nommée par un vocabulaire précis s'inspirant du principe de la nomenclature : les machines Conord possèdent une « cuve en émail-porcelaine inoxydable", un "batteur aluminium", "un essorage par rouleaux » et " une vidange à pompe centrifuge ${ }^{19}$ tandis que la machine CLEM doit son succès à sa « turbine compound brevetée $»^{20}$.

La référence à cet univers est à mettre en relation avec le contexte de développement décrit plus en avant et qui marque les années 1950 de ses effets favorables sur l'économie. Elle fait, d'ailleurs, écho à la fascination plus générale qu'exercent la technique et le progrès scientifique à cette période, qualifiée positivement, par la rédaction de Marie-Claire, d' « ère atomique " :

«Car l'âge atomique, c'est aussi l'âge de l'abondance, de l'émancipation, du progrès social, l'âge des maisons claires, des enfants bien portants, du réfrigérateur, du lait pasteurisé, de la machine à laver, l'âge du confort, de la qualité et du bon marchée1. " 
Le rapprochement du travail domestique et de la sphère professionnelle passe également par la création d'images montrant les femmes en activité (ménage, soin du linge, cuisine) et en tenue de travail. Le tablier qu'elles portent est une protection à enfiler au dessus des vêtements lors d'une activité jugée salissante. Il est, à ce titre, revêtu dans l'exercice de nombreux métiers. Il en existe plusieurs types dont l'usage varie en fonction des domaines professionnels : celui du boucher n'est pas le même que celui du serveur ou de la femme de chambre (Pellegrin, 2009). Sur la publicité pour le Reluiseul Johnson diffusée en octobre 1954, dans la troisième vignette, la ménagère nettoie le sol à l'aide d'un balai. Sur sa robe à pois, un tablier blanc, dit tablier à bavette, permet de protéger l'ensemble de sa tenue dans son activité de nettoyage. Un peu plus loin, dans le même numéro, sur la publicité pour le savon Le Chat, la femme porte un tablier sans bavette, qui rappelle celui des femmes de chambre. Dans le contexte du magazine, ces images sont interprétées en regard du discours qui l'accompagne et qui a déjà été évoqué : le tablier est un vêtement de protection et un vêtement de travail.

Image 4. Page 20, Publicité Johnson, Marie-Claire, n 1, octobre 1954

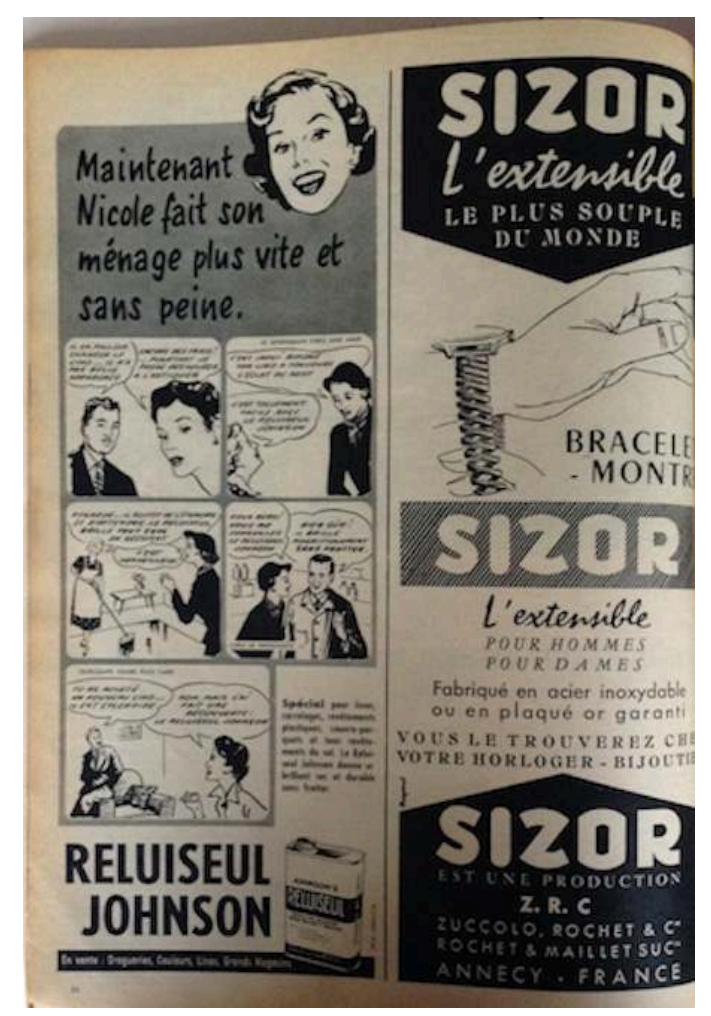

Coll. Musée Galliera 
Image 5. Page 34, Publicité Le Chat, Marie-Claire, nº 1, octobre 1954

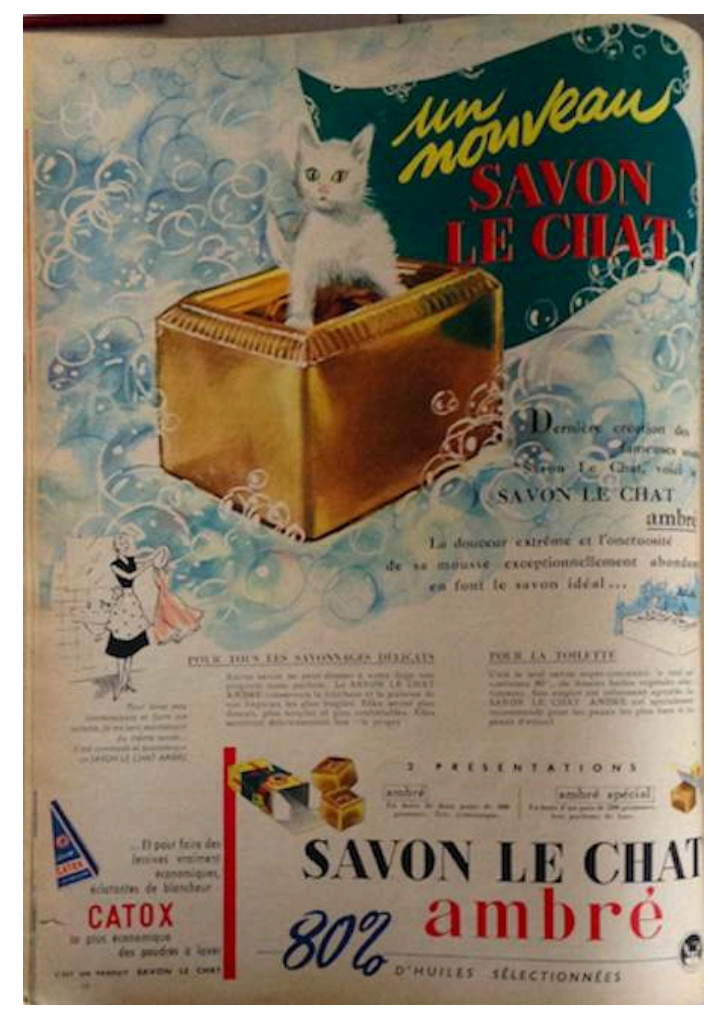

Coll. Musée Galliera

Cette formule visuelle, qualifiée ainsi parce qu'elle est répétée à plusieurs reprises, dans le contenu éditorial ${ }^{22}$ et publicitaire, n'est pas identifiable à la seule présence d'un tablier-uniforme. Ce dernier est aussi mobilisé comme une pièce de mode valorisant certaines parties du corps de ces femmes et par là un corps genré (Pellegrin, 2009). Dans la publicité pour le tissu Rhonyl publiée en avril 1955, la ceinture du tablier, très ajustée fait apparaître une taille exagérément fine, la bavette, en cœur, souligne la forme des seins tandis que le port des talons qui accentue la finesse des chevilles sert le jeu de jambes. La position, bras levés de cette ménagère, renforce ce corps aux formes marquées jusqu'à l'irréalisme. Ce schéma de représentation, repris du dessin de mode, est également présent dans la publicité pour la gazinière Gaz, publiée dans le même numéro. 
Image 6. Page 13, Publicité Rhonyl, Marie-Claire, n 7, avril 1955

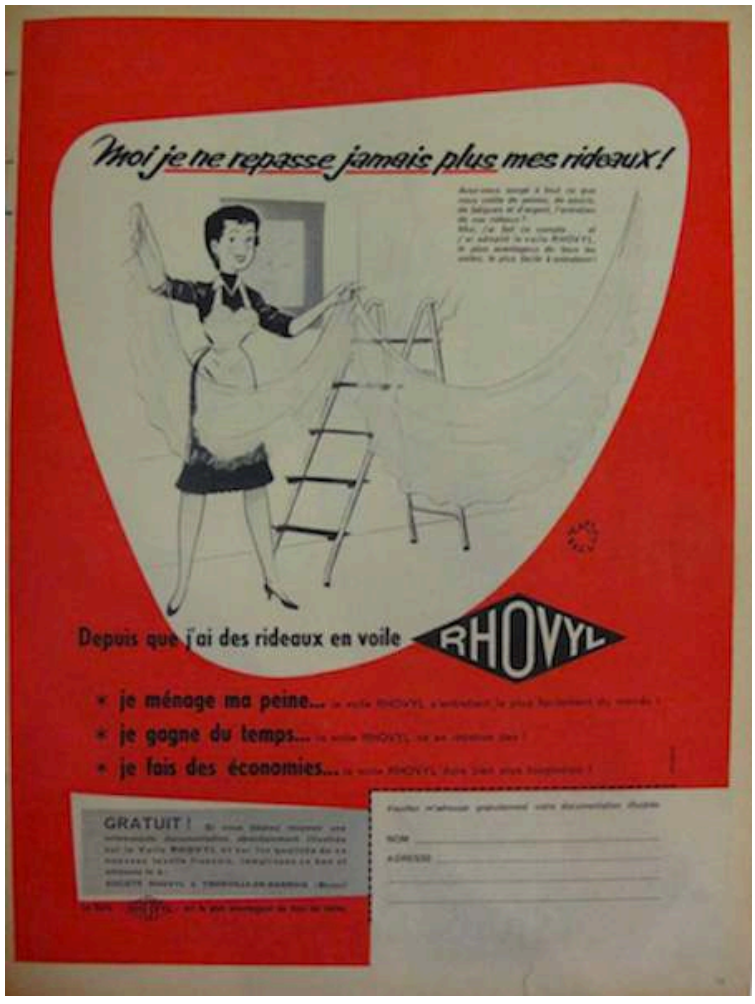

Coll. Musée Galliera

Image 7. Page 9, Publicité Gaz, Marie-Claire, nº 7, avril 1955

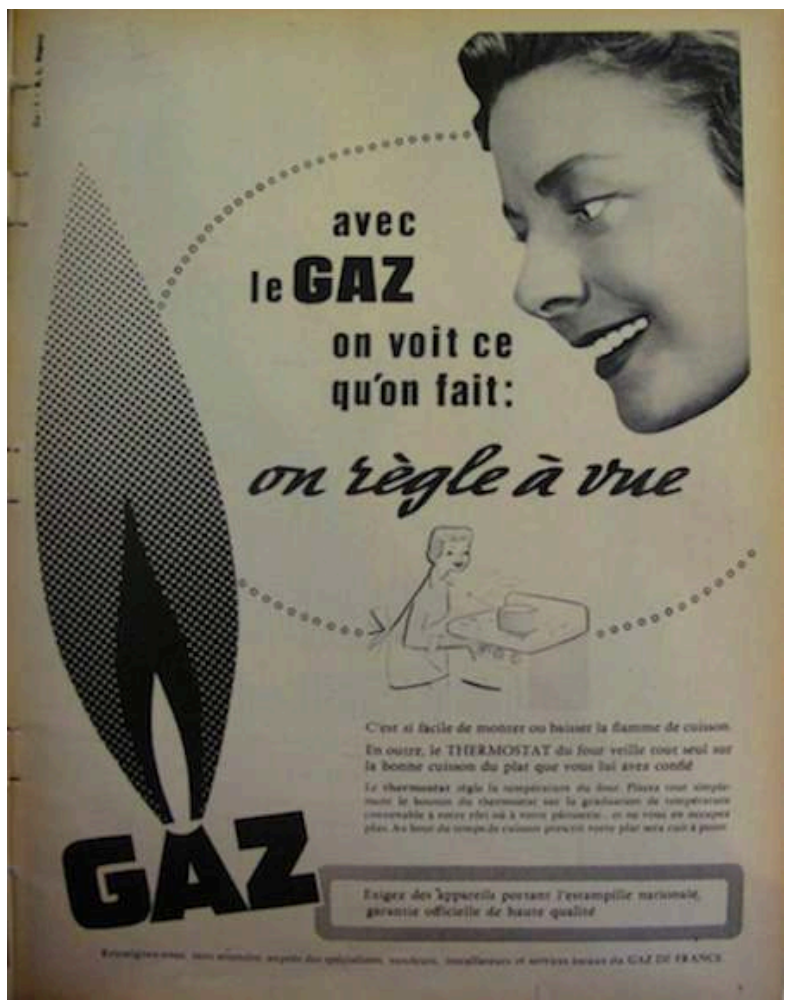

Coll. Musée Galliera 
Occupant moins de place dans la page que dans l'exemple précédent, la femme est dessinée dans un trait relativement simple. Quelques éléments synthétisent l'idée globale: le ruban noué dans le dos aide à identifier le tablier et marque taille et poitrine. Cette référence à la valorisation d'un corps vêtu pour séduire, est d'ailleurs évoquée dans les textes, notamment dans les publicités pour les tabliers.

Image 8. Page 39, publicité Lavaclor, Marie-Claire, n², novembre 1954

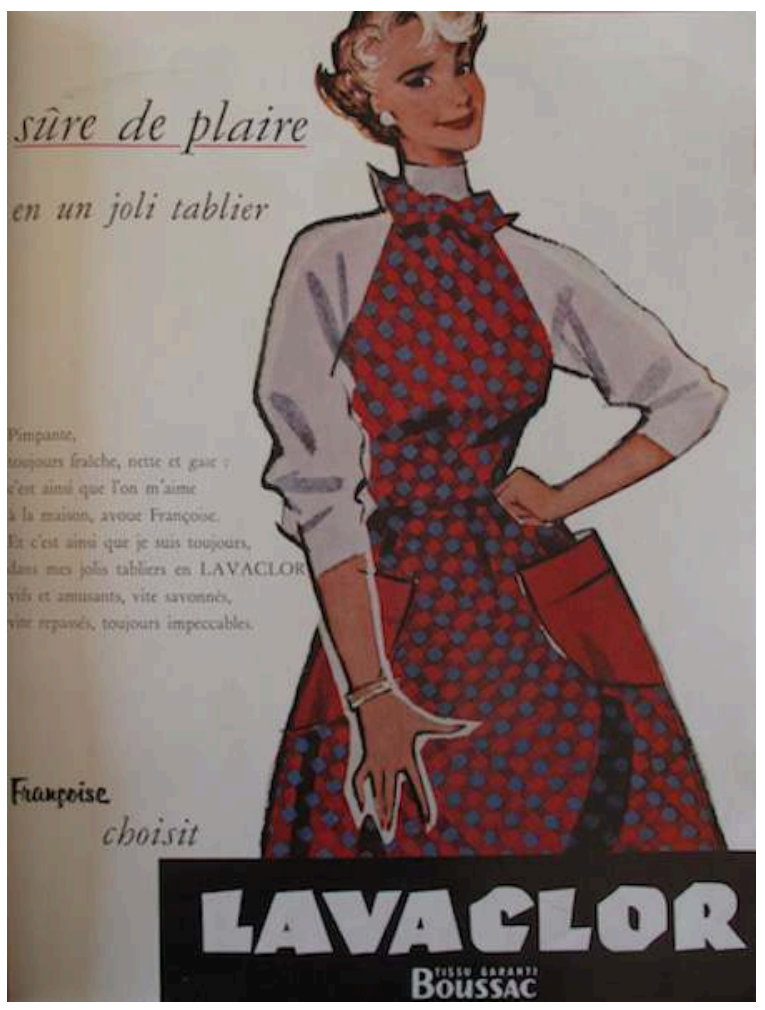

Coll. Part.

Dans celle pour Lavaclor, publiée en novembre 1954, l'argument de la séduction est associé à celui de la praticité :

"Sûre de plaire en un joli tablier. Pimpante, toujours fraîche, nette et gaie : c'est ainsi que l'on m'aime à la maison, avoue Françoise. Et c'est ainsi que je suis toujours, dans mes jolis tabliers en Lavaclor vifs et amusants, vite savonnés, vite repassés, toujours impeccables ${ }^{23}$.»

Cette formule visuelle n'est que peu réaliste si l'on considère la difficulté d'accomplir les tâches domestiques en portant une robe ajustée et des talons hauts tout en restant ainsi apprêtée. Cette figure est une illustration au sens où elle met en image une idée préétablie (Gunthert, 2010). Son irréalisme fait apparaître la construction d'un discours narratif et visuel qui ne fait pas forcément référence à la réalité des pratiques mais à la symbolique qu'elle signifie : le travail domestique envisagé comme un métier valorisant et la nécessité pour les femmes de garder une apparence séduisante en toutes circonstances. 


\section{Conclusion}

Ainsi, on peut dire qu'un magazine est un objet de divertissement dont la rentabilité repose sur l'attrait qu'il exerce auprès des annonceurs qui cherchent à promouvoir la production industrielle auprès d'un lectorat visé et donc de la capacité qu'a la rédaction à intéresser les lectrices. De ce fait, le contenu diffusé par le magazine implique d'être cohérent avec les attentes des lectrices. Le développement du modèle de féminité fondé sur la gestion du foyer développé dans Marie-Claire à partir de 1954 indique qu'il fait sens pour les lectrices issues des classes moyennes auxquelles il est adressé.

Celui-ci est composé de plusieurs facettes lisibles à travers l'important développement narratif mis en œuvre, abordant des domaines aussi variés que l'aménagement du logement, l'alimentation de la famille, l'éducation des enfants et la gestion du budget familial mais également dans le travail des images composées à partir de registres visuels divers évoquant le monde industriel, professionnel ou des apparences qui permet de synthétiser les différentes facettes de ce modèle tout en renforçant les aspects symboliques les plus saillants: les femmes ont un rôle dans la modernisation des pratiques domestiques, dans l'ascension sociale des familles mais aussi dans le maintien des rapports de séduction au sein du couple.

Les images médiatiques, comme celles étudiées ici, doivent être mises en relation avec les discours qu'elles participent à construire, condition nécessaire pour en faire des sources signifiantes. Le corpus d'images étudiées ici ne permet pas de participer à écrire l'histoire du travail des femmes mais en revanche, il offre des pistes de réflexion pour une histoire des représentations de genre au sein desquelles le monde du travail est mobilisé et où une analyse des rôles genrés a tout sa place.

\section{BIBLIOGRAPHIE}

Bard C. (2003), "Les reines du foyer dans l'entre-deux guerres », Les femmes dans la société française au 20e siècle, Paris, Armand Colin, p. 48.

Eck H., Blandin C. (2010), La vie des femmes. La presse féminine aux XIXe et XXe siècles, Paris, Éditions Panthéon-Assas.

Clarke J. (2005), « L'organisation ménagère comme pédagogie », Travail, genre et sociétés, $\mathrm{n}^{\circ} 13$, p. $139-157$.

Cott N. F. (2002), « La femme moderne. Le style américain dans les années vingt. », in Thébaud F. (dir.), V. Le XXe siècle, dans Duby Georges, Perrot Michelle (dir.), Histoire des femmes en Occident, Paris, Perrin, p. 145-164.

Delaunay Q. (2003), Société industrielle et travail domestique : l'électroménager en France, XIX-XXe siècle, Paris, L'Harmattan.

Geers A (2016), Le sourire et le tablier. La construction médiatique du féminin dans Marie-Claire de 1937 à nos jours, thèse de doctorat, EHESS. 
Gunthert A. (2010), «L'illustration, ou comment faire de la photographie un signe », L'Atelier des icônes, [En ligne], mis en ligne le 12 octobre 2010, consulté le 18 décembre 2017. URL : http:// culturevisuelle.org/icones/1147

Kergoat D. (2005), «12. Rapports sociaux et division du travail entre les sexes », Femmes, genre et sociétés, Paris, La Découverte, «TAP/Hors Série ». URL : www.cairn.info/femmes-genre-etsocietes--9782707144126-page-94.htm

Knibiehler Y. (1997), La Révolution maternelle. Femmes, maternité, citoyenneté depuis 1945, Paris, Perrin.

Leymonerie C. (2006), « Le Salon des arts ménagers dans les années 1950. Théâtre d'une conversion à la consommation de masse ", Vingtième Siècle, nº 91, p. 43-56.

Martin M. (1987), « Ménagère : une profession ? Les dilemmes de l'entre-deux-guerres », Le Mouvement Social, n 140, p. 89-106.

Martin J. (1998), « Politique familiale et travail des femmes mariées en France. Perspective historique : 1942-1982 », Population, n 6, p. 1119-1154. URL : http://www.persee.fr/web/revues/ home/prescript/article/pop_00324663_1998_num_53_6_6960.

Pellegrin N. (2009), Tabliers au masculin, tabliers au féminin. Catalogue de l'exposition des Musées de la ville de Chauvigny (Vienne), juillet-décembre 2009, Donjon de Gouzon, Chauvigny, France, Association des publications chauvinoises.

Segalen M. et Le Wita B. (1993), « Les vertus de l'intérieur », in Segalen Martine et Le Wita Béatrix, (dir.), Chez soi : objets et décors : des créations familiales ?, Paris, Autrement, p. 30-50.

Thébaud F. (2001), « Féminisme et maternité », in Yvonne Knibiehler (dir.), Maternité, affaire privée, affaire publique, Paris, Bayard.

Werner F. (1984), « Du ménage à l'art ménager : l'évolution du travail ménager et son écho dans la presse féminine de 1919 à 1939 ", Le Mouvement Social, n 129, p. 61-87.

Woronoff D. (1998), Histoire de l'industrie en France du XVIe à nos jours, Paris, Seuil.

\section{NOTES}

1. «Éditorial », Marie-Claire, n 1, 5 mars 1937, p. 5.

2. La rationalisation domestique est une gestion des tâches ménagères calquée sur celle du travail industriel, héritée des principes du taylorisme, qui vise à améliorer la gestuelle et l'aménagement de l'espace pour gagner du temps (Clarke, 2005).

3. À la même époque, on retrouve aux USA la diffusion d'un modèle proche (Cott, 2002).

4. « Dictionnaire pratique pour enlever toutes les taches », Marie-Claire, $n^{\circ} 29,17$ septembre 1937, p. 36.

5. « De ravissantes coiffeuses avec une table en bois blanc », Marie-Claire, $n^{\circ} 27,3$ septembre 1937, p. 28.

6. «Promenez-vous le dimanche et le lundi, ne mourrez pas de faim. Ce qu'il faut toujours avoir en réserve ", Marie-Claire, $n^{\circ}$ 9, 30 avril 1937, p. 34 ; « Les bons desserts », Marie-Claire, $\mathrm{n}^{\circ}$ 38, 12 novembre 1937, p. 34 ; «La friture », Rubrique La bonne cuisine, Marie-Claire, n 3, 19 mars 1937, p. 46.

7. Marie-Claire, $\mathrm{n}^{\circ}$ 1, octobre 1954, p. 110-111.

8. « L'Équipe 55 », Marie-Claire, n 4, janvier 1955, p. 108-117.

9. Marie-Claire, $n^{\circ} 2$, novembre 1954, p. 52-53. 
10. Marie-Claire, $\mathrm{n}^{\circ}$ 2, novembre 1954, p. 120-121.

11. "Pour vos demi-pensionnaires, préparez chaque soir un savoureux cocktail de vitamines ", Marie-Claire, $\mathrm{n}^{\circ}$ 2, novembre 1954, p. 60-61.

12. Rubrique «Claude Maman », Marie-Claire, $n^{\circ}$ 5, février 1955, p. 52-53.

13. Publicité Lu, Marie-Claire, $n^{\circ} 5$, février 1955, p. 9.

14. Publicité Royco, Marie-Claire, $n^{\circ} 5$, février 1955, p. 43.

15. «Ne laissez pas votre enfants devenir un inconnu », Marie-Claire, $n^{\circ} 1$, octobre 1954, p. 42-43.

16. «Le crédit vous permet », Marie-Claire, $\mathrm{n}^{\circ}$ 1, octobre 1954, p. 116-117.

17. «Le crédit vous permet », Marie-Claire, $n^{\circ} 1$, octobre 1954, p. 116-117.

18. Publicité CLEM, Marie-Claire, $n^{\circ}$ 2, novembre 1954, p. 104 et Publicité Frigidaire, Marie-Claire, n 3, décembre 1954, p. 113.

19. Publicité Conord, Marie-Claire, $\mathrm{n}^{\circ} 2$, novembre 1954, p. 54.

20. Publicité CLEM, Marie-Claire, $\mathrm{n}^{\circ}$ 2, novembre 1954, p. 104.

21. «L'Équipe 54 entre chez vous », Marie-Claire, n 1, octobre 1954, p. 110-111.

22. Illustration en vignettes de l'article « Le crédit vous permet ", Marie-Claire, $\mathrm{n}^{\circ}$ 1, octobre 1954, p. 116-117.

23. Publicité Lavaclor, Marie-Claire, $\mathrm{n}^{\circ}$ 2, novembre 1954, p. 39.

\section{RÉSUMÉS}

Cet article porte sur la construction narrative, visuelle et symbolique d'un récit médiatique adressé aux lectrices de Marie-Claire. À partir de 1954, rédaction et publicitaires proposent une nouvelle image de la féminité, en s'attachant au rôle des femmes dans le foyer qu'ils décrivent comme un métier basé sur des connaissances techniques. Ces images rappellent plusieurs univers visuels : le travail, avec la reprise des codes du dessin industriel ou le tablier comme référence au vêtement de travail ainsi que la mode, symbolisant en filigrane la nécessité pour les femmes d'être séduisantes en toutes circonstances. Ce modèle de féminité permet de vendre les produits (mobilier en série, électroménager, alimentation industrielle, etc) fabriqués par les différentes industries qui fleurissent après-guerre et qui cherchent à toucher des consommatrices de plus en plus nombreuses. Il valorise et ancre, dans la modernité, un rôle de genre qui donne aux épouses et mères des classes moyennes une responsabilité dans l'ascension sociale de la famille et dans la refondation de la société française d'après-guerre.

This article focuses on the narrative, visual and symbolic construction of a media report addressed to or intended for Marie-Claire's readers. From 1954, editorials and advertisers offer a new image of femininity, focusing on the role of women in their home they describe as a profession based on technical knowledges or skills. These images remind us of several visual universes: work, with the use of industrial design codes, or the apron as a reference to workwear and fashion, symbolizing the need for women to be attractive in all circumstances. This model of femininity makes it possible to sell products (mass-produced furnitures, household appliances, industrial food, etc.) made by the various industries that flourish after the war and that seek to reach more and more consumers. It values and anchors, in modernity, a gender role that gives middle-class wives and mothers a responsibility in the social rise of the family and in the refoundation of post-war French society. 
INDEX

Mots-clés : genre, magazine féminin, foyer, représentations médiatiques

Keywords : gender, women's magazine, household, media framing

\section{AUTEUR}

\section{ALEXIE GEERS}

Alexie Geers est docteure en histoire visuelle (EHESS) et chercheuse associée au CEREP/

Université de Reims. Sa thèse est intitulée "Le sourire et le tablier. La construction médiatique du féminin dans Marie-Claire de 1937 à nos jours." Ses recherches portent sur l'histoire des images médiatiques genrées, sur les pratiques médiaculturelles des femmes et sur la transmission des savoirs de genre 